\title{
CO AND NEAR-INFRARED OBSERVATIONS OF HIGH-REDSHIFT SUBMILLIMETER GALAXIES
}

\author{
D. T. FRAYER \\ California Institute of Technology \\ Astronomy Dept. 105-24 \\ Pasadena, CA 91125 \\ E-mail:dtf@astro.caltech.edu \\ UMass/INAOE Conference on \\ Deep Millimeter Surveys: Implications for Galaxy Formation and Evolution \\ 19-21 June 2000
}

\begin{abstract}
I discuss our ongoing Owens Valley Millimeter Array observations and Keck nearinfrared wavelength observations of the high-redshift sub-mm population of galaxies. These observations are important for our understanding of the distant universe since the sub-mm population accounts for a large fraction of the extragalactic background at $\mathrm{mm} / \mathrm{sub}-\mathrm{mm}$ wavelengths and contributes significantly to the total amount of star-formation and AGN activity at high redshift. The CO data suggest that the sub-mm galaxies are analogous to the gas-rich ultraluminous systems found in the local universe. Initial near-infrared data show that many of the sub-mm galaxies are faint-red sources which are undetected at ultraviolet/optical wavelengths. These results highlight the importance that future sensitive mmwavelength instruments, such as the LMT and ALMA, will have on our understanding of the early evolution and formation of galaxies.
\end{abstract}

\section{Introduction}

The discovery of an ultraluminous population of high-redshift galaxies with deep submillimeter sufveys has revolutionized our understanding of the distant universe.25.2.19.12. The current data show that the sub-mm population has a mixture of AGN and starburst characteristics with properties which are roughly consistent with the local population of ultraluminous $\left(L>10^{12} L_{\odot}\right)$ infrared galaxies (ULIGs). The relative importance of AGN and starburst activity in powering the high luminosities of the sub-mm population is still an open question, but the growing consensus is that the majority of the luminosity of the population is powered by star formation $\mathrm{O}$ The early $\mathrm{CO}$ and $\mathrm{X}$-ray data on the sub-mm population support the starburst nature of the population by showing the presence of sufficient molecular gas to fuel the star-formation activity, 14 and the lack of expected X-ray emission if mostly dominated by AGN $13,28.24$.

Although the redshift distribution of the sub-mm population is still uncer- 
Table 1. Brightest Sources in SCUBA Cluster Lens Survey

\begin{tabular}{|c|c|c|c|c|}
\hline Galaxy & $\underset{(\mathrm{mJy})}{\mathrm{S}(850 \mu \mathrm{m})}$ & Redshift & $K-\operatorname{mag}$ & Notes \\
\hline SMM J02399-0136 & 25.4 & 2.8081 .5 & 19.1 且 & Col15 \\
\hline SMM J00266+1708 & 18.6 & $>2.0$ 28 & 22.516 & \\
\hline SMM J09429+4658 & 17.2 & $>3.928$ & 19.4 글 & ERA \\
\hline SMM J14009+0252 & 14.5 & $>0.7$ 28 & $21.0 \stackrel{20}{=}$ & $\mathrm{J} 5 \stackrel{20}{=}$ \\
\hline SMM J14011+0252 & 12.3 & 2.56514 & 17.8 & $\mathrm{CO}^{14}$ \\
\hline SMM J02399-0134 & 11.0 & 1.062 29 & $16.3 \mathrm{29}$ & $\mathrm{CO}_{22}$ \\
\hline SMM J22471-0206 & 9.2 & $>1.8$ & ? & \\
\hline SMM J02400-0134 & 7.6 & $>2.4 \stackrel{28}{=}$ & $?$ & \\
\hline SMM J04431+0210 & 7.2 & $>1.6 \mathrm{2}$ & $19.1 \mathrm{k7}$ & ERO-N427 \\
\hline
\end{tabular}

tain, the majority of the sub=mm galaxies are believed to be at high redshifts $(z \gtrsim 2)$ based on their radid 188 and near-infrared 27 data. The early redshift distributions basedon optical imaging and spectroscopy suggested somewhat lower redshifts, 26323 but several of the original candidate optical counterparts have turned out to be incorrect. Despite their ultra-high luminosities, many sub-mm galaxies are undetected at ultraviolet/optical wavelengths de to extinction by dust. For these highly obscured galaxies, follow-up radides and/or mm interferometric observations $10,16,6,17$ are required in order to uncover the proper counter-part.

In order to understand the nature of the sub-mm population, we have been carrying out multi-wavelength observations of individual systems in the SCUBA Cluster Lens Survey 26 This survey represents sensitive sub-mm mapping of seven massive, lensing clusters which uncovered 15 background sub$\mathrm{mm}$ sources. The advantages of this sample are that the amplification of the background sources allows for deeper source frame observations and that lensing by cluster potentials does not suffer from differential lensing. We have concentrated our efforts on the nine background galaxies detected at the highest signal-to-noise (Table 1). Only three sources have spectroscopic redshifts, and the redshift Jower limits shown in Table 1 are based on their sub-mm/radio flux ratios 28

\section{CO Results}

At OVRO we have conclusively detected CO emission from two sub-mm systems, SMM J02399-0136 at $z=2.8$ (SMM J02399) and SMM J14011+0252 

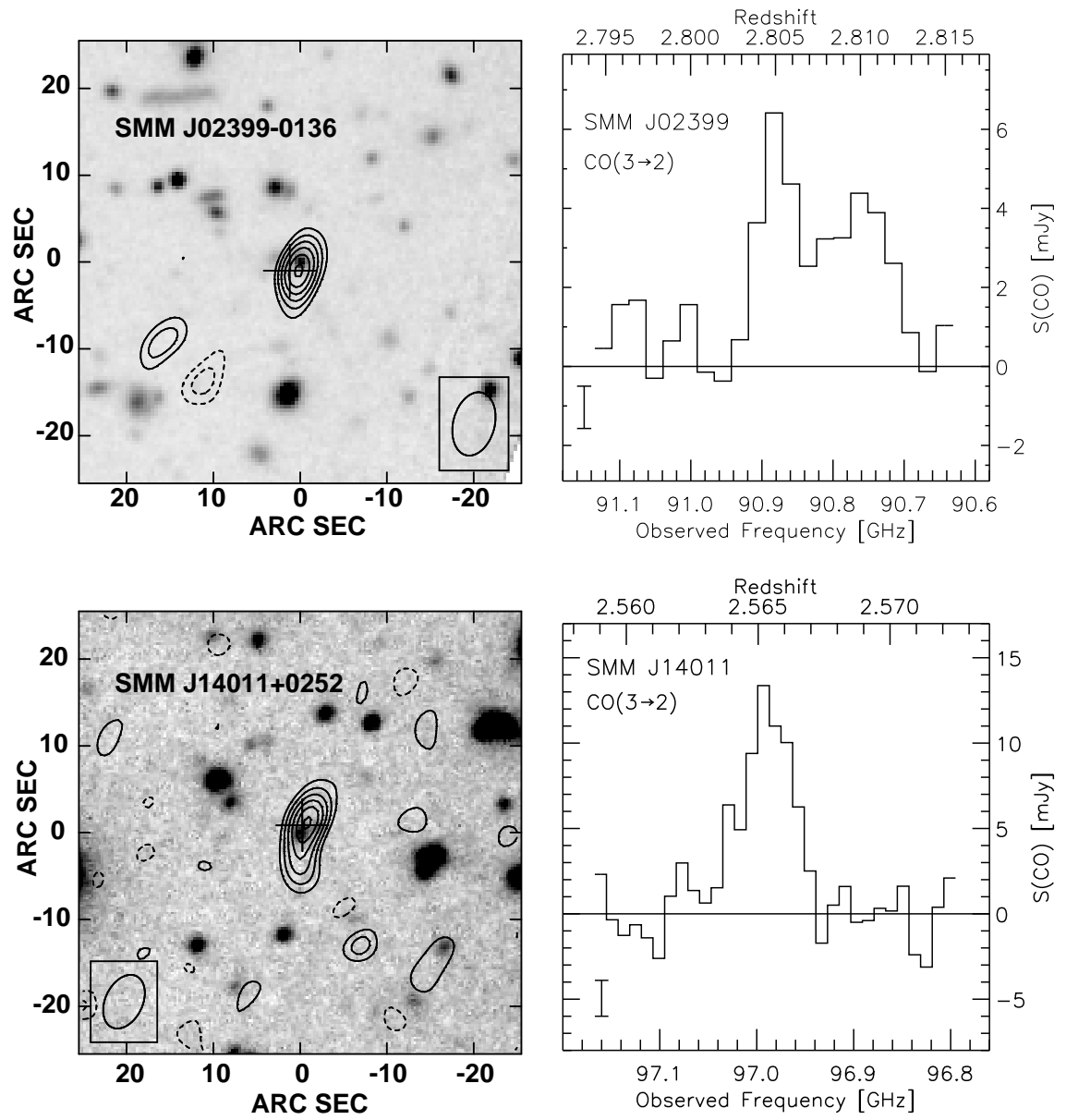

Figure 1. OVRO CO(3-2) detections for SMM J02399-0136 (top) and SMM J14011+0252 (bottom). The grey-scale images at the left are optical images while the contours represent the integrated CO maps for the sub-mm galaxies. The crosses represent the positions of the SCUBA detection. The corresponding $\mathrm{CO}(3-2)$ spectra are shown at the right. These data were originally published in Frayer et al. $(1998,1999)$.

at $z=2.6$ (SMM J14011) (Fig. 1). A third system SMM J02399-0134, which is associated with a ring-galaxy containing a Seyfert nucleus at $z=1.06$, 29 has recently been detected in $\mathrm{CO}$ at the PdB.22 We have also tentatively con- 
firmed the PdB detection at OVRO. To date, these three galaxies are the only sub-mm sources with known redshifts, and it is promising that all three have already been detected in $\mathrm{CO}$. The early $\mathrm{CO}$ results suggest that the sub-mm population contains massive reservoirs of molecular gas and are among the most $\mathrm{CO}$ luminous galaxies in the universe.

The strongest sub-mm source, SMM J02399, shows an AGN component in its optical spectrum,21 while SMM.14011 shows only evidence for starburst activity at optical/NIR wavelengths 20 Although the optical characteristics of these two galaxies are vastly different, their radio, sub-mm, and CO properties are fairly similar and are consistent with a high level of star formation activity (SFRs of a few $\times 10^{2} \mathrm{M}_{\odot} \mathrm{yr}^{-1}$ to more than $10^{3} \mathrm{M}_{\odot} \mathrm{yr}^{-1}$, depending on the IMF and AGN contamination). After correcting for lensing, we derive CO luminosities of $3-4 \times 10^{10} \mathrm{~K} \mathrm{~km} \mathrm{~s}^{-1} \mathrm{pc}^{2}\left(\mathrm{H}_{o}=50 \mathrm{~km} \mathrm{~s}^{-1} \mathrm{Mpc}^{-1} ; q_{o}=1 / 2\right)$ in these two systems. These $\mathrm{CO}$ luminosities correspond to molecular gas masses of about $5 \times 10^{10}-2 \times 10^{11} \mathrm{M}_{\odot}$, depending on the exact value of the $\mathrm{CO}$ to $\mathrm{H}_{2}$ conversion factor. Both SMM J02399 and SMM J14011 appear to be associated with a merger event. Given that mergers of gas-rich galaxies at low-redshift result in massive starbursts, we expect star-formation to be an important component for powering the far-infrared luminosities in both of these systems. In fact, the large molecular gas masses of SMM J02399 and SMM J14011 are sufficient to form the stars of an entire $\mathrm{L}^{*}$ galaxy, which suggests that the sub-mm population may represent the formative phase of massive galaxies.

SMM J02399 is unresolved in CO, while SMMJ14011 is extended over a large spatial scale in its source frame $(\gtrsim 10 \mathrm{kpc})$. Figure 1 contains low resolution OVRO data which showed tentative evidence for extended CO emission in the north-south direction in SMM J14011. The extended morphology of the molecular gas in SMMJ14011 has been recently confirmed with higher-resolution data from OVRO and BIMA. These results may suggest that SMM J14011 is in an early stage of its merger event, unlike the majority of ULIGs in the local universe whose CO emission is mostly contained within the central kpc.11 It is currently unknown what fraction of the sub-mm sources are compact or are extended over large spatial scales as is SMM J14011. If the progenitors of the sub-mm systems are more gas rich than those of local ULIGs, we could expect the sub-mm sources to have larger gas fractions and to be more extended than their low-redshift analogs. A large sample of sub-mm sources need to be observed in CO before statistical comparisons could be made between the CO properties of local ULIGs and the high-redshift sub-mm galaxies.

Conference proceedings to be published by World Scientific 


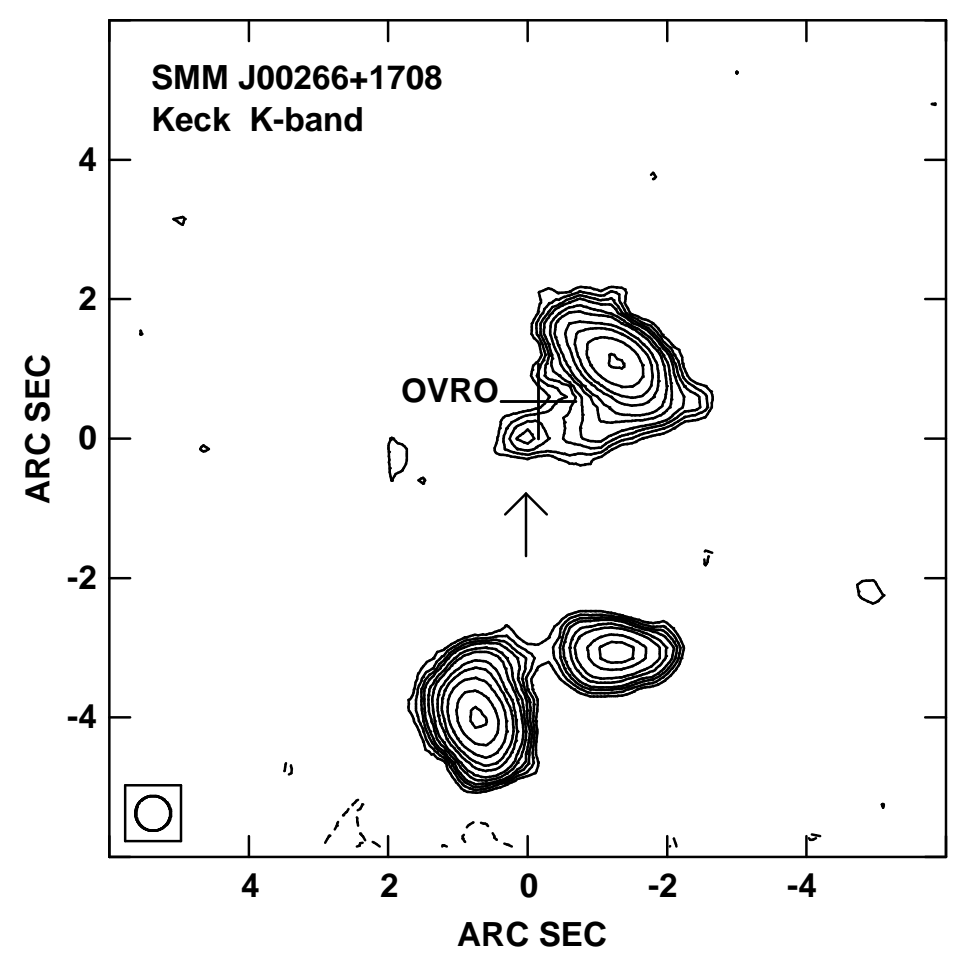

Figure 2. The Keck $K$-band $(2.2 \mu \mathrm{m})$ image of SMM J00266+1708. The near-infrared data was taken after determining the accurate position of the sub-mm source with OVRO $1.3 \mathrm{~mm}$ imaging. The position of the $1.3 \mathrm{~mm}$ source is shown by the cross labeled "OVRO". The three bright sources in the field are foreground galaxies previously observed at optical wavelengths. The arrow points to the new galaxy not detected in the optical $(I>26)$ thought to be the counter-part of SMM J00266+1708. The rms of the image is $24.8 \mathrm{mag} / \mathrm{sq}-$ $\operatorname{arcsec}(0.04 \mu \mathrm{Jy} /$ beam $)$, and the contours are $1 \sigma \times(-3,3,4,5,6,8,10,15,20,30,50,80)$. The seeing disk (beam) of the near-infrared data is shown in the lower left $\left(0.5 \times 0.5^{\prime \prime}\right)$. These data were originally published in Frayer et al. (2000).

\section{$3 \quad$ Near-Infrared Results}

Many sub-mm galaxies are too obscured by dust to be detected at ultraviolet/optical wavelengths. At least four of the nine sources in our sample which were undetected at optical wavelengths $(I>25-26)$ have faint nearinfrared, $K$-band counter-parts. Two of these are bright enough in $K$-band 
( $K=19.1,19.6 \mathrm{mag})$ to be classified as extremely red objects (EROs) 27 An additional faint $(K=21)$ galaxy was found associated with a relatively bright $(0.5 \mathrm{mJy})$ radio counter-part 20 The fourth and faintest galaxy with a near-infrared counter-part is SMM J00266+1708.

The sub-mm position of SMM J00266+1708 is located between three optically visible galaxies.26 We imaged the field at OVRO at $1.3 \mathrm{~mm}$ and determined its position to be offset from all three optical sources. Deep, follow-up near-infrared observations with NIRC on Keck uncovered a new faint galaxy at $K=22.5$ located at the position of the $1.3 \mathrm{~mm}$ source 10 Although SMM J00266+1708 is the second brightest sub-mm source in the SCUBA Cluster Lens Survey, it is currently the faintest known near-infrared counter-part of a sub-mm galaxy discovered to date (Fig. 2).

Only two of the nine sources in the sample still require deep $K$ band imaging and currently have uncertain counter-parts. The galaxy SMM J02400-0134 has no optically detected galaxies near the sub-mm position. For SMM J22471-0206 there are several optical galaxies which could be the sub-mm counter-part, but given previous results it will be interesting to test whether or not any new candidate galaxies are uncovered with deep $K$ band imaging. Depending on the results for these last two unknown systems, the current data suggest that approximately $40 \%-70 \%(4 / 9-6 / 9)$ of the sub$\mathrm{mm}$ population as a whole have faint near-infrared counter-parts which are undetected at optical wavelengths. Only $30 \%-40 \%(3 / 9-4 / 9)$ of the sample have optical counterparts ( $I<26-27$, correcting for source lensing).

The $K$-band magnitudes of the sub-mm counter-parts in the SCUBA Cluster Lens Survey range over 6 magnitudes which reflects the wide diversity of colors and redshifts for the population (Table 1). The magnitudes listed in Table 1 have not been corrected for lensing; unlensed sources would be about a magnitude fainter on average. Currently, only the three brightest optical ( $I$-band) sources have spectroscopic redshifts. The other sources are much fainter and would require near-infrared spectroscopy to obtain redshifts, which will be challenging even with $8 \mathrm{~m} / 10 \mathrm{~m}$ class ground base telescopes. Our early spectroscopic results with NIRSPEC on Keck suggest that lines may be detectable in the brighter sources $(K \lesssim 20)$, while many of the fainter sub-mm sources $(K \gtrsim 22)$ may have to wait for the Next Generation Space Telescope.

\section{Conclusions}

Most sub-mm sources are too red and/or faint to be detected at optical wavelengths. There is very little overlap, if any, between the ultraluminous sub-mm population and the less luminous, optically-selected Lyman Break population 
of galaxies. This highlights the importance of radio, millimeter, and nearinfrared observations of the sub-mm population for our general understanding of the evolution and formation of galaxies.

Potentially, we do not need to wait for future optical/near-infrared spacebased missions to obtain redshifts for the bulk of the sub-mm population. Redshifts could be determined directly from the $\mathrm{CO}$ lines themselves with planned ground-based millimeter telescopes, such as the LMT and ALMA. Both ALMA and the LMT will have sufficient sensitivities and broad-bandwidth spectrometer capabilities to make large $\mathrm{CO}$ redshift surveys practical 5 The proposed $30 \mathrm{GHz}$ spectrometer for the LMT would be an excellent redshift machine for the sub-mm population of galaxies.

The two best studied sub-mm galaxies (SMM J02399-0136 and SMM J14011+0252) share many of the same properties of the local population of ULIGs, such as high infrared luminosities, the association with mergers, massive molecular gas reservoirs, comparable CO line widths, and similar IR/radio and IR/CO luminosity ratios. Future CO observations of large samples of ultraluminous galaxies with ALMA and the LMT will enable us to study the evolution of the molecular gas properties as a function of redshift which will be crucial for our understanding of the star-formation history of the universe.

\section{Acknowledgments}

I thank the work of my collaborators Nick Scoville, Rob Ivison, Ian Smail, Andrew Blain, Aaron Evans, Min Yun, and Jean-Paul Kneib. I appreciate the efforts of the OVRO and Keck staff who have made these observations a success. I acknowledge support from NSF grant AST 9981546 made to the OVRO Millimeter Array which is operated by the California Institute of Technology. I thank the organizers at the University of Massachusetts and INAOE for planning the conference and providing support to attend the conference.

\section{References}

1. Barger, A. J., Cowie, L. L., Mushotzky, R. F., \& Richards, E. A. 2000, AJ submitted (astro-ph/0007175)

2. Barger, A. J., Cowie, L. L., Sanders, D. B., Fulton, E., Taniguchi, Y., Sato, Y., Kawara, K., \& Okuda, H. 1998, Nature, 394, 248

3. Barger, A. J., Cowie, L. L., Smail, I., Ivison, R. J., Blain, A. W., \& Kneib, J.-P. 1999, AJ, 117, 2656 
4. Bertoldi, F., et al. 2000, A\&A, in press astro-ph/0006094

5. Blain, A. W., Frayer, D. T., Bock, J. J., \& Scoville, N. Z. 2000, MNRAS, 313,559

6. Blain, A. W., Kneib, J.-P., Ivison, R. J., \& Smail, I. 1999a, ApJ, 512, L87

7. Blain, A. W., Smail, I., Ivison, R. J., \& Kneib, J.-P. 1999b, MNRAS, 302, 632

8. Bryant, P. M., \& Scoville, N. Z. 1999, AJ, 117, 2632

9. Carilli, C. L., \& Yun, M. S. 1999, ApJ, 513, L13

10. Downes, D., et al. 1999, A\&A, 347, 809

11. Downes, D., \& Solomon, P. M. 1998, ApJ, 507, 615

12. Eales, S., Lilly, S., Gear, W., Dunne, L., Bond, J. R., Hammer, F., Le Fèvre, O., \& Crampton, D. 1999, ApJ, 515, 518

13. Fabian, A. C., et al. 2000, MNRAS, 315, L8

14. Frayer, D. T., et al. 1999, ApJ, 514, L13

15. Frayer, D. T., Ivison, R., J., Scoville, N. Z., Yun, M., Evans, A. S., Smail, I., Blain, A. W., \&, Kneib, J.-P. 1998, ApJ, 506, L7

16. Frayer, D. T., Smail, I., Ivison, R. J., \& Scoville, N. Z. 2000, AJ, in press (astro-ph/0005239)

17. Gear, W. K., Lilly, S. J., Stevens, J. A., Clements, D. L., Webb, T. M., Eales, S. A., \& Dunne, L. 2000, MNRAS, in press (astro-ph/0007054)

18. Hornschemeier, A. E., et al. 2000, ApJ, in press astro-ph/0004260)

19. Hughes, D., et al. 1998, Nature, 394, 241

20. Ivison, R. J., Smail, I., Barger, A. J., Kneib, J.-P., Blain, A. W., Owen, F. N., Kerr, T. H., \& Cowie, L. L. 2000, MNRAS, 315, 209

21. Ivison, R. J., Smail, I., Le Borgne, J.-F., Blain, A. W., Kneib, J.-P., Bézecourt, J., Kerr, T. H., \& Davies, J. K. 1998, MNRAS, 298, 583

22. Kneib, J.-P., et al. 2000, in preparation

23. Lilly, S. J., Eales, S., A., Gear, W. K. P., Hammer, F., Le Fèvre, O., Crampton, D., Bond, J. R., \& Dunne, L. 1999, ApJ, 518, 641

24. Severgnini, P., et al. 2000, A\&A, in press (astro-ph/000623)

25. Smail, I., Ivison, R. J., \& Blain, A. W. 1997, ApJ, 490, L5

26. Smail, I., Ivison, R. J., Blain, A. W., \& Kneib, J.-P. 1998, ApJ, 507, L21

27. Smail, I., Ivison, R. J., Kneib, J.-P., Cowie, L. L., Blain, A. W., Barger, A. J., Owen, F. N., \& Morrison, G. 1999, MNRAS, 308, 1061

28. Smail, I., Ivison, R. J., Owen, F. N., Blain, A. W., \& Kneib, J.-P. 2000, ApJ, 528, 612

29. Soucail, G., Kneib, J. P., Bézecourt, J., Metcalfe, L., Altieri, B., Le Borgne, J. F. 1999, A\&A, 343, L70 